\title{
Clinical Trial Highlights: Phase III Study in Spotlight
}

\author{
Kevin McFarthing \\ Parkinson's Advocate, Innovation Fixer Ltd, Oxford, UK \\ kevin(dot)mcfarthing(at)googlemail(dot)com
}

Tanya Simuni

Parkinson's Disease and Movement Disorders Center,

Northwestern University Feinberg School of Medicine, Chicago, USA

TITLE: A Phase 3 study of isradipine as a disease modifying agent in patients with early Parkinson's disease (STEADY-PD III):

STATUS: Study completed, results pending.

CLINICALTRIALS.GOV ID: NCT02168842.

SPONSOR: NINDS.

ENROLMENT: 336 .

ESTIMATED COMPLETION DATE: Winter 2019.

OBJECTIVE: To assess the efficacy of isradipine, a dihydropyridine calcium channel antagonist to slow the progression of Parkinson's disease (PD).

BACKGROUND: Isradipine, a dihydropyridine calcium channel antagonist (DHP) that is approved for the treatment of hypertension, is being tested as a potential disease modifying intervention in early PD. Isradipine was shown to be neuroprotective in in vitro and in vivo models of parkinsonism[1]. The mechanism of neuroprotection is linked to selective vulnerability of substantia nigra pars compacta neurons that preferentially express L-type calcium channels. Neuroprotective effects of isradipine are achieved at a plasma concentration that is obtained within the safe dose range for human administration and consistent with the tolerable dosage identified in the phase II study of isradipine in PD (STEADY-PDII)[2]. A number of epidemiological studies have demonstrated a reduced risk of development of PD in individuals treated with DHPs compared to other antihypertensive agents.

STUDY DESIGN and OUTCOMES: STEADY-PD III is an NINDS funded Phase 3, parallel group, placebocontrolled 36 months study evaluating the efficacy of isradipine 10mg daily as a disease-modifying agent in early PD[3]. The study is being conducted at 54 Parkinson Study Group sites in US and Canada. The study recruited 336 participants with de novo PD not requiring symptomatic therapy and followed them prospectively for 36 months. The primary outcome is the change from baseline in the Unified Parkinson Disease Rating Scale (UPDRS) Part I-III score as measured in the ON state at month 36, in the active arm compared to the placebo arm. Secondary outcome measures include: 1) Time to initiation and utilization of dopaminergic therapy; 2) Time to onset of motor complications; 3) Change in non-motor disability and other PD motor and non-motor outcome measures

CURENT STUDY STATUS: Enrolment was started in November 2014 and was completed in 12 months, 6 months ahead of schedule, including $10 \%$ minority recruitment. The last partiicpant completed the study in November 2018. Study retention rate is 95\%, 297 have initiated PD symptomatic therapy. Data lock is scheduled for January 2019 and final data analysis will be available February 2019. 


\section{Comments:}

STEADY-PD III final results will be presented at the American Academy of Neurology May 2019 meeting. The study has a number of unique design features, including the longest duuration disease modifying interventional study (3-year) in a de novo PD population and assessment of the primary outcome in the medication ON state. Retention and completion rates have been higher than expected for such a long duration study.

\section{REFERENCES:}

1. Chan, C.S., et al., 'Rejuvenation' protects neurons in mouse models of Parkinson's disease. Nature, 2007. 447(7148): p. 1081-6.

2. Phase II safety, tolerability, and dose selection study of isradipine as a potential disease-modifying intervention in early Parkinson's disease (STEADY-PD). Mov Disord, 2013.

3. Biglan, K.M., et al., A novel design of a Phase III trial of isradipine in early Parkinson disease (STEADYPD III). Ann Clin Transl Neurol, 2017. 4(6): p. 360-368. 